\title{
ACURÁCIA DE TESTES FUNCIONAIS NA IDENTIFICAÇÃO DA PRÉ-ATIVAÇÃO DE MÚSCULOS LOMBOPÉLVICOS
}

\author{
ACCURACY OF FUNCTIONAL TESTS FOR IDENTIFYING ANTICIPATORY ACTIVATION \\ OF THE LUMBOPELVIC MUSCLES
}

Artigo Original

Original ARTICLE

Artículo Original

\author{
PRECISIÓN DE PRUEBAS FUNCIONALES EN LA IDENTIFICACIÓN DE LA PRE-ACTIVACIÓN \\ DE LOS MÚSCULOS LUMBOPÉLVICOS
}

\begin{abstract}
Fabrício José Jassi' (Fisioterapêuta) Tiago Del Antonio' (Fisioterapêuta) Raphael Gonçalves de Oliveira' (Educador Físico)

Laís Campos de Oliveira' (Fisioterapêuta)

Neri Alves² (Educador Físico)

Fábio Mícolis de Azevedo ${ }^{2}$

(Fisioterapêuta)

Thaís Cristina Chaves ${ }^{3}$

(Fisioterapêuta)

Rúben de Faria Negrão Filho ${ }^{2}$ (Fisioterapêuta)

1. Universidade Estadual do Norte do Paraná - UENP, Jacarezinho, PR, Brasil.

2. Universidade Estadual Paulista "Júlio de Mesquita Filho" (UNESP), Faculdade de Ciência e Tecnologia, Departamento de Física, Química e Biologia, Presidente Prudente, SP, Brasil.

3. Faculdade de Medicina de Ribeirão Preto da Universidade de São Paulo, FMRP-USP, Departamento de Neurociências e Ciências do Comportamento, Ribeirão Preto, SP, Brasil.
\end{abstract}

\section{Correspondência:}

Alameda Padre Magno, 841, Bairro Nova Alcântara, Jacarezinho, PR, Brasil. 86400-000.

fjjassi@uenp.edu.br

\section{RESUMO}

Introdução: Ajustes antecipatórios (pré-ativação) dos músculos profundos do tronco aumentam a estabilidade lombopélvica. Estudos prévios demonstram que indivíduos assintomáticos podem apresentar atrasos no início de ativação muscular e alterações físico-funcionais. No entanto, não foram encontrados estudos que tenham verificado se testes físico-funcionais (TFF) são capazes de identificar alteração no início de ativação dos músculos estabilizadores lombopélvicos. Objetivo: Verificar os níveis de sensibilidade, especificidade e acurácia de um conjunto de testes de avaliação da capacidade física funcional para detectar alterações na pré-ativação dos músculos transverso do abdome/oblíquo interno (TrA/OI) e multífido lombar (ML) durante o teste de movimento rápido de flexão do ombro (TMRFO). Método: Participaram do estudo 27 voluntários assintomáticos para dor lombar, com média de idade de 23,8 anos (desvio padrão: 2,2), e que foram submetidos aos TFF e TMRFO para determinação do início da ativação dos músculos profundos do tronco por meio da eletromiografia de superfície. Foi verificada sensibilidade e especificidade e análise da acurácia através da curva ROC (Receiver Operating Characteristic) e teste de qui-quadrado para comparações entre porcentagens $(p<0,05)$. Resultados: A "pré-ativação" foi a condição que ocorreu com maior frequência no grupo como um todo, bem como em ambos os gêneros, com exceção do TrA/OI no gênero feminino $\left(\chi^{2}=0,28 / P=0,58\right)$. Entre todos os TFF aplicados, o de enrolamento repetitivo do tronco apresentou maior valor de sensibilidade, especificidade e área sob a curva $\operatorname{ROC}(0,75 ; 0,73 ; 0,74$, respectivamente). Conclusão: Entre os TFF avaliados, o de enrolamento repetitivo do tronco mostrou níveis aceitáveis de acurácia para identificar alterações na ativação da musculatura estabilizadora lombopélvica em voluntários assintomáticos para dor lombar. Assim, o teste de enrolamento repetitivo do tronco pode ser utilizado na clínica para predizer alterações na ativação dos músculos profundos do tronco.

Descritores: eletromiografia; estabilização; aptidão física; região lombossacral.

\section{ABSTRACT}

Introduction: Anticipatory adjustments (pre-activation) of the deep trunk muscles increase lumbopelvic stability. Previous studies have shown that asymptomatic individuals may experience delays in the start of muscle activation, as well as functional physical alterations. However, there are no studies that assess whether physical and functional tests (PFT) are able to identify changes in early activation of the lumbopelvic stabilizer muscles. Objective: To assess the levels of sensitivity, specificity and accuracy of a set of physical and functional performance tests to detect changes in anticipatory activation of the transversus abdominis/internal oblique muscles (TrA/OI) and lumbar multifidus (ML) during a task of fast shoulder flexion movements (FSFM). Method: Twenty-seven volunteers, asymptomatic for low back pain, with a mean age of 23.8 (Standard deviation:2.2) years, participated in the study. They were submitted to PFT and FSFM to determine the initial activation time of the deep trunk muscles through surface electromyography. Sensitivity and specificity were determined, and accuracy was analyzed by the ROC (Receiver Operating Characteristic) curve and chi-square test for comparisons between percentages $(p<0.05)$. Results: Anticipatory activation was the condition that occurred most frequently in the group as a whole, and in both genders, with the exception of TrA $/ O$ in females $\left(\chi^{2}=0.28 / P=0.58\right)$. Among all the PFT assessed, the repetitive trunk flexion-extension test showed the highest values for sensitivity, specificity and area under the ROC curve $(0.75 ; 0.73 ; 0.74$, respectively). Conclusion: Among the PFT evaluated, the repetitive trunk flexion-extension test showed acceptable levels of accuracy for identifying changes in the activation of deep trunk muscles in volunteers who were asymptomatic for low back pain. Thus, the repetitive trunk flexion-extension test can be used clinically to predict changes in the activation of deep muscles of the trunk.

Keywords: electromyography; stabilization; physical fitness; lumbosacral region.

\section{RESUMEN}

Introducción: Ajustes de anticipación (pre-activación) de los músculos profundos del tronco aumentan la estabilidad lumbopélvica. Estudios anteriores demostraron que individuos asintomáticos (sin dolor) pueden presentar 
retrasos en el inicio de la activación muscular y alteraciones físicas y funcionales. Sin embargo, no fueron encontrados estudios que hayan verificado si las pruebas físicas y funcionales (PFF) son capaces de identificar los cambios en la activación temprana de los músculos estabilizadores lumbopélvicos. Objetivo: Verificar los niveles de sensibilidad, especificidad y exactitud de un conjunto de pruebas de evaluación de la capacidad física funcional para detectar cambios en la pre-activación de los músculos transverso abdominal/oblicuo interno (TrA/Ol) y multífido lumbar (ML) durante la prueba de movimiento rápido de flexión del hombro (PMRFH). Método: Participaron del estudio 27 voluntarios, asintomáticos para el dolor lumbar, con una edad media de 23,8 años (desviación estándar: 2,2), sometidos a PFF y PMRFH para determinar el inicio de la activación de los músculos profundos del tronco mediante electromiografía de superficie. Se encontró una sensibilidad y especificidad y análisis de exactitud a través de la curva ROC (Receiver Operating Characteristic) y la prueba de chi-cuadrado para las comparaciones entre porcentajes ( $p<$ 0,05). Resultados: La "pre-activación" fue la condición que ocurrió con más frecuencia en el grupo como un todo y en ambos sexos, con excepción de TrAvOl en las mujeres $\left(\chi^{2}=0,28 / P=0,58\right)$. Entre todos los PFF aplicados, la flexión repetitiva del tronco presentó un mayor valor de sensibilidad, especificidad y el área bajo la curva $R O C(0,75 ; 0,73$; 0,74, respectivamente). Conclusión: Entre los PFF evaluados, I flexión repetitiva del tronco mostró niveles aceptables de exactitud para identificar cambios en la activación de los músculos estabilizadores lumbopélvicos en voluntarios asintomáticos para dolor lumbar. Así, la prueba de flexión repetitiva del tronco se puede utilizar clínicamente para predecir cambios en la activación de los músculos profundos del tronco.

Descriptores: electromiografía; estabilización; aptitud física; región lumbosacra.

\section{INTRODUÇÃO}

Tem-se verificado que alguns músculos do tronco especialmente o transverso do abdômen/oblíquo interno (TrA/OI) e multífidos lombar (ML) apresentam ação muscular antecipatória (pré-ativação) para estabilizar a região lombo-pélvica ${ }^{1-3}$. O Sistema Nervoso Central (SNC) prevendo mudanças na configuração corporal em decorrência do movimento faz com que estes músculos sejam pré-ativados de forma a minimizar as sobrecargas nas estruturas responsáveis pela estabilidade articular ${ }^{4,5}$.

Para avaliar a pré-ativação muscular vem sendo utilizada a Eletromiografia de Superfície (EMGS) durante a tarefa do movimento rápido do membro superior, considerando como pré-ativação qualquer início de atividade dos músculos do tronco que ocorram 100ms antes ou até $50 \mathrm{~ms}$ após a ativação inicial do músculo deltóide ${ }^{5,6}$. A literatura aponta que indivíduos com dor lombar tendem a apresentar alteração no tempo de início de ativação muscular? , entretanto, indivíduos assintomáticos também podem apresentar tal alteração na pré-ativação8,9. Esses aspectos sugerem que a pré-ativação pode ser utilizada como parâmetro de alteração na estabilidade lombo-pélvica em sujeitos com dor lombar ou se apresentar como fator preditivo para o desenvolvimento de dor lombar ${ }^{8}$ em indivíduos assintomáticos.

Além disso, os Testes Físicos Funcionais (TFF) também podem fornecer parâmetros sobre estabilidade lombo-pélvica, já que indivíduos com dor lombar tendem a apresentar comprometimento na capacidade física e funcional. Algumas dessas alterações são: hipotrofia muscular, menor resistência e força muscular ${ }^{10-12}$, déficit proprioceptivo $^{13}$, alterações de extensibilidade muscular e no controle motor ${ }^{10,14}$. Para avaliar a capacidade físico-funcional da região lombo-pélvica, diferentes testes podem ser encontrados na literatura ${ }^{15-17}$. Esses TFF podem avaliar coordenação e equilíbrio, encurtamento muscular, resistência, comprimento e força, bem como, a qualidade dos movimentos.

Considerando que tanto a pré-ativação quanto os testes físicos funcionais podem ser utilizados como parâmetros de estabilização lombo-pélvica, não foram encontrados estudos que tenham verificado se testes físico-funcional são capazes de identificar alterações na ativação dos músculos estabilizadores lombo-pélvicos. Níveis adequados de sensibilidade, especificidade e acurácia dos TFF de estabilidade lombo-pélvica podem identificar sinais de alterações na pré-ativação dessa musculatura. Assim, é possível que testes clínicos físico-funcionais comumente utilizados possam ser utilizados para avaliação e acompanhamento de indivíduos assintomáticos, porém em risco de desenvolver dor lombar.

Portanto, o objetivo deste trabalho foi verificar os níveis de sensibilidade, especificidade e acurácia de um conjunto de testes de avaliação de capacidade físico-funcional em detectar alterações na pré-ativação dos músculos TrA/OI e ML durante o teste de movimento rápido de flexão do ombro (TMRFO), bem como, verificar diferenças na frequência de pré-ativação ou alterações na pré-ativação para os músculos TrA/OI e ML entre os gêneros. Nossa hipótese central foi de que níveis adequados de sensibilidade, especificidade e acurácia seriam observados para os TFF na detecção de pré-ativação dos músculos estabilizadores lombo-pélvicos em sujeitos assintomáticos.

\section{MATERIAIS E MÉTODOS}

Esse estudo observacional transversal foi aprovado pelo Comitê de Ética em Pesquisa da FCT/UNESP - Campus de Presidente Prudente (parecer $n^{\circ}$ 255/2008).

Para realização deste estudo foram selecionados 27 voluntários assintomáticos para dor lombar, estudantes universitários de ambos os gêneros, sendo 15 mulheres e 12 homens com idades entre $20 \mathrm{e}$ 28 anos (idade: $23.8 \pm 2,2$; peso: $62,6 \pm 11.0$; altura: 1,65 0,2; índice de massa corporal (IMC) $21,8 \pm 2,3$. Os critérios de elegibilidade foram: IMC entre 18,5 a $24,9 \mathrm{~kg} / \mathrm{m}^{2}$, ausência de dor lombar nos últimos seis meses, doenças osteomioarticulares e neurológicas. Como critérios de exclusão: voluntários submetidos a tratamento para dor lombar ou procedimento cirúrgico nesta região, dor no ombro que impedisse a flexão do ombro, presença de alterações posturais severamente incapacitantes e gestação para mulheres.

Para garantir a seleção dos voluntários com relação à ausência de dor lombar e alterações posturais, todos responderam a um questionário de incapacidade relacionada à dor lombar ${ }^{18}$ e foram submetidos a um procedimento para classificação postural ${ }^{19}$. Após a triagem os voluntários foram apresentados aos procedimentos da pesquisa e assinaram o Termo de Consentimento Livre e Esclarecido.

Os voluntários foram submetidos a dois procedimentos de avaliação 
realizados em dias diferentes (com intervalo de 24 horas). Os Testes Físico-Funcionais (TFF) para mensurar a capacidade física e funcional para estabilização lombo-pélvica foram avaliados na sessão 1 de avaliação e o Teste de Movimento Rápido de Flexão do Ombro (TMRFO) para determinação do início de ativação dos músculos ML e TrA/OI por meio de EMG-S foi realizado na sessão 2 de avaliação (24 horas após a realização da sessão 1).

\section{Testes Físico-Funcionais (TFFs)}

Os TFFs utilizados neste estudo foram testes clínicos relacionados com a estabilidade lombo-pélvica, organizados em quatro domínios, avaliados na seguinte ordem: (1) coordenação e equilíbrio, (2) extensibilidade, (3) resistência e (4) comprimento e força. Todo o procedimento de coleta referente ao TFF foi realizado por um avaliador com experiência clínica e constou dos seguintes testes: 1) Teste de Coordenação e Equilíbrio em apoio unipodal com os olhos fechados ${ }^{15}$; 2) Testes de Extensibilidade - foi aplicado o teste de Thomas modificado ${ }^{15}$ para avaliar o comprimento do íliopsoas e reto femoral (bilateral) e o teste de Schober modificado ${ }^{16}$ para avaliar a extensibilidade muscular da coluna lombar; 3) Testes de Resistência - foram aplicados quatro testes baseados nos trabalhos de Cox $^{15}$ e Liebenson ${ }^{16}$, sendo o teste de agachamento repetitivo - para avaliar o quadríceps e o glúteo máximo, o de enrolamento repetitivo do tronco - para avaliar a resistência dinâmica dos flexores do tronco, o de resistência estática dos músculos extensores das costas (teste de Sorensen), e o teste de ponte lateral - que avalia a resistência estática dos oblíquos e quadrado lombar e 4) Teste de Comprimento e Força, sendo avaliados os músculos psoas, fibras posteriores do glúteo médio e glúteo máximo (bilateral) ${ }^{16}$. Todos os testes foram realizados sempre por um mesmo examinador treinado para realização dos testes e obtenção dos dados.

Cada voluntário foi submetido ao conjunto de testes em um único dia, com intervalo de dois minutos entre cada teste e todos os valores obtidos durante os testes foram registrados e classificados como normal ou alterado.

\section{Instrumentação}

Para captação dos sinais EMGS foram utilizados pares de eletrodos de superfície, modelo Meditrace da marca Kendall', com superfície de captação de $10 \mathrm{~mm}$ de diâmetro, separados entre si por 20mm. Após tricotomia e assepsia da pele com álcool, os eletrodos foram posicionados sobre os músculos ML e DA (SENIAN $\left.{ }^{20}\right)$ e sobre o TrA/OI $(2 \mathrm{~cm}$ mediais e inferiores a espinha ilíaca ântero-superior ${ }^{21}$ ).

Os eletrodos foram conectados a um circuito pré-amplificador com ganho de 20 vezes, Common Mode Rejection Ratio (CMRR) maior que 80 dB e impedância de $1012 \Omega$ e ao módulo condicionador de sinais analógicos (da marca EMG System do Brasi ${ }^{\circledR}$ ) no qual foram digitalmente filtrados com passa-faixa de $20 \mathrm{~Hz}$ e $500 \mathrm{~Hz}$ e amplificados para um ganho final de 1000 vezes. A placa de conversão de sinal analógico para digital (A/D) foi configurada para frequência de amostragem de $1000 \mathrm{~Hz}$.

\section{Teste de movimento rápido de flexão do ombro}

Os voluntários foram orientados a permanecer na posição ortostática, pernas estendidas, pés mantidos separados a uma distância correspondente à largura do quadril e braços relaxados e pendentes ao lado do corpo. O movimento foi executado com o braço dominante, sendo que os voluntários foram instruídos e treinados a realizar flexão do ombro com velocidade máxima e amplitude de aproximadamente $60^{\circ}$ (com cotovelo em posição de máxima extensão), imediatamente após o estímulo visual22. Desta forma foram captados sinais EMGS dos músculos ML, TrA/OI e DA (Deltoide Anterior) de 12 movimentos de flexão do ombro.

\section{Processamento dos dados (EMG-S)}

Os dados foram selecionados e exportados para o Matlab ${ }^{\text {Tm}}$ Versão 6.5 para processamento e determinação do tempo de início de ativação muscular do DA, TrA/OI e ML. Desta forma, na rotina desenvolvida no Matlab, os traçados eletromiográficos selecionados foram inicialmente submetidos à retificação total do sinal, envoltória linear com filtro passa baixa de $50 \mathrm{~Hz}$ (Butterworth de 6a ordem). Em seguida, o algoritmo apresentou uma janela, na tela do Matlab ${ }^{\text {Tm}}$, para identificação do início da ativação que foi determinada considerando-se o ponto no qual o sinal desviou da linha de base mais do que três desvios-padrão, por um tempo mínimo de $25 \mathrm{~ms}^{23}$.

O tempo de início de ativação dos três músculos foi obtido simultaneamente. Dessa forma, após a determinação do início de ativação muscular dos três músculos (TrA/OI, ML e DA) a rotina determinava a diferença entre os tempos obtidos para os músculos TrA/OI e ML em relação ao DA. Esse procedimento foi adotado considerando-se que o músculo DA é o principal agonista do movimento de flexão do ombro (movimento solicitado durante o teste). Dessa forma, sua ativação foi utilizada como referência para determinação da existência de pré-ativação ou não dos músculos analisados - TrA/OI e ML (músculos que realizam a estabilização do tronco para que o movimento do braço ocorra).

Para análise dos valores de atividade eletromiográfica obtidos optou-se por classificar os participantes em duas condições "pré-ativação" e "alteração da pré-ativação". A primeira condição denominada "pré-ativação" se referiu a valores obtidos dentro do tempo considerado como adequado para pré-ativação $(-100 \mathrm{~ms}$ antes da ativação do DA na flexão de ombro e 50ms após início de ativação do DA na flexão de ombro). Na segunda condição considerada como "alteração da pré-ativação" foram considerados aqueles valores no qual o tempo de início de ativação estava fora do previsto como pré-ativação, ou não ocorreu pré-ativação (Figura 1).

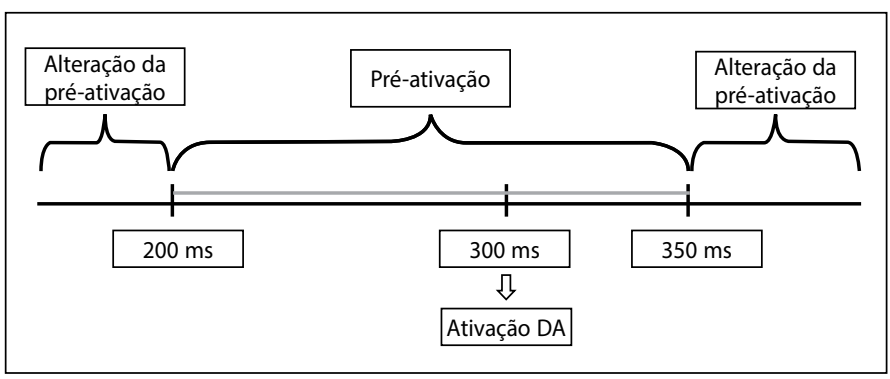

Figura 1. Esquema ilustrativo exemplificando a rotina utilizada para identificação do tempo de ativação dos músculos Transverso/obliquo interno (TrA/OI) e Multífidos $(\mathrm{ML})$ em relação ao músculo deltoide anterior (DA). Qualquer ativação no período entre -100ms antes da ativação do músculo DA e 50ms após a ativação do músculo DA foi considerada como pré-ativação. No exemplo acima, o músculo DA foi ativado em 300 ms, então qualquer início de ativação 100 ms anterior (200 ms) ou 50 ms posterior (350 ms) foi considerada como pré-ativação. Início de ativação fora deste intervalo foi considerado como "alteração da pré-ativação".

\section{Análise estatística}

Para analisar a sensibilidade (\% de indivíduos com alteração nos TFF/\% de indivíduos com alteração da pré-ativação) e especificidade (\% de indivíduos sem alteração nos TFF/\% de indivíduos sem alteração da pré-ativação) dos TFF com relação ao resultado da EMGS (considerado como padrão-ouro) foi utilizada a análise da curva ROC (Receiver Operating Characteristic) e Area Under de Curve (AUC) por meio do software Origin ${ }^{\text {Tm }}$ versão 8.0. Desta forma, a variável teste (TFF) foi testada com relação ao maior valor de sensibilidade, especificidade e área sob a curva na tentativa de discriminar o resultado da variável de estado (pré-ativação dos músculos estabilizadores lombo-pélvicos 
através da EMGS). A classificação adotada para interpretação dos valores obtidos para a área sob a curva ROC foram os seguintes: área>0,9: alta acurácia, 0,7-0,9: acurácia moderada, 0,5-0,7: baixa acurácia e 0,5: resultado ao acaso ${ }^{24}$

Para verificar diferenças entre as porcentagens foi utilizado o teste de qui-quadrado $\left(c^{2}, p \leq 0.05\right)$ (SPSS versão 22, IBM SPSS Statistics for Windows, Version 22.0. Armonk, NY: IBM Corp, 2013).

\section{RESULTADOS}

\section{Testes físico-funcionais}

De todos os TFF, aqueles com maior número de voluntários com alterações foram: Teste de Thomas modificado (18 voluntários $=66,66 \%$ ), teste de Schober modificado (14 voluntários=51,85\%), Teste de enrolamento repetitivo do tronco ( 11 voluntários=40,74\%), Teste estático de resistência das Costas de Sorensen (15 voluntários $=55,55 \%)$ e o Teste de comprimento e força para o glúteo máximo (23 voluntários=85,18\%). Apenas para o teste de comprimento e força para o glúteo máximo foi observada diferença significativa de indivíduos com alteração em relação ao número de participantes sem alteração no teste $\left(c^{2}(1, N=27)=6, P=0,01\right)$.

\section{EMGS}

A porcentagem de indivíduos classificados nas condições de "pré-ativação" ou "alteração da pré-ativação" para os músculos ML (fibras superficiais) e TrA/OI obtidos durante o TMRFO está descrita na Tabela 1. Os valores foram apresentados de acordo com a frequência de ocorrência das repetições realizadas no teste ( $n=12$ repetições/voluntário) (Tabela 1).

De acordo com os valores demonstrados na Tabela 1, pode-se observar que a presença de "pré-ativação" foi a condição que ocorreu com maior frequência no grupo como um todo, bem como em ambos os gêneros para os músculos avaliados, com exceção do TrA/OI no gênero feminino.

Considerando-se as diferenças entre os gêneros, foi observada frequência significativamente maior de "alteração da pré-ativação" para o ML e TrA/OI no gênero feminino (Tabela 1). Não foram verificadas diferenças nas porcentagens da categoria de "pré-ativação" entre os gêneros (Tabela 1).

\section{Testes físico-funcionais e EMGS}

Todos os valores dos TFF para detectar as alterações na pré-ativação através da EMGS foram então submetidos à análise de acurácia através da curva ROC bem como sensibilidade e especificidade (Tabela 2).

Os testes que apresentaram área sob a curva ROC próximo ou acima de 0,7 (acurácia moderada) e demonstraram os maiores valores de sensibilidade e/ou especificidade foram: testes de Coordenação e Equilíbrio do membro inferior esquerdo ( $A \cup C=0,69$; sensibilidade $=0,50$ e especificidade $=0,89$ ) e Enrolamento Repetitivo do Tronco ( $A \cup C=0,74$; sensibilidade $=0,75$ e especificidade $=0,73$ ), Comprimento (Tabela 2 e Figura 2).
Tabela 2. Resultados obtidos da curva ROC (EMG-S X Testes Físicos Funcionais).

\begin{tabular}{|c|c|c|c|c|}
\hline ROC curve & Área & $95 \%$ IC & Sensibilidade & Especificidade \\
\hline \multicolumn{5}{|c|}{ Coordenação e Equilíbrio } \\
\hline Ml-d & 0,59 & $0,37-0,82$ & 0,25 & 0,95 \\
\hline Ml-e & 0,69 & $0,49-0,90$ & 0,50 & 0,89 \\
\hline \multicolumn{5}{|c|}{ Encurtamento muscular } \\
\hline$P-d$ & 0,50 & $0,26-0,75$ & 0,12 & 0,89 \\
\hline P-e & 0,54 & $0,30-0,78$ & 0,25 & 0,84 \\
\hline RF-d & 0,41 & $0,16-0,65$ & 0,25 & 0,57 \\
\hline RF-e & 0,39 & $0,15-0,63$ & 0,37 & 0,42 \\
\hline Espinhais Lombares & 0,54 & $0,30-0,79$ & 0,62 & 0,47 \\
\hline \multicolumn{5}{|c|}{ Resistência } \\
\hline $\begin{array}{l}\text { Agachamento } \\
\text { repetitivo }\end{array}$ & 0,53 & $0,29-0,77$ & 0,12 & 0,94 \\
\hline $\begin{array}{c}\text { Enrolamento } \\
\text { repetitivo do tronco }\end{array}$ & 0,74 & $0,53-0,95$ & 0,75 & 0,73 \\
\hline $\begin{array}{l}\text { Estático de resistência } \\
\text { das costas de sorensen }\end{array}$ & 0,39 & $0,15-0,63$ & 0,37 & 0,42 \\
\hline $\begin{array}{c}\text { Estático de resistência } \\
\text { do quadrado lombar } \\
\text { (direito) }\end{array}$ & 0,49 & $0,24-0,73$ & 0,25 & 0,73 \\
\hline $\begin{array}{c}\begin{array}{c}\text { Estático de resistência } \\
\text { do quadrado lombar } \\
\text { (esquerdo) }\end{array} \\
\end{array}$ & 0,51 & $0,27-0,76$ & 0,25 & 0,79 \\
\hline
\end{tabular}

\begin{tabular}{c|c|c|c|c}
\hline \multicolumn{5}{c}{ Comprimento e Força } \\
\hline P-d & 0,68 & $0,48-0,88$ & 0,37 & 1,00 \\
\hline P-e & 0,60 & $0,38-0,83$ & 0,37 & 0,84 \\
\hline Gm-d & 0,54 & $0,30-0,78$ & 0,25 & 0,84 \\
\hline Gm-e & 0,54 & $0,30-0,78$ & 0,25 & 0,84 \\
\hline GM-d & 0,60 & $0,35-0,85$ & 1,00 & 0,21 \\
\hline GM-e & 0,68 & $0,43-0,93$ & 1,00 & 0,36 \\
\hline
\end{tabular}

Nota: MI- $d$ = Membro Inferior direito; MI-e = Membro Inferior esquerdo; $P$ - $d=$ Psoas direito; P-e = Psoas esquerdo; RF- $d$ = Reto Femoral direito; RF-e = Reto Femoral esquerdo; $G m-d$ = Glúteo médio direito; Gm-e = Glúteo médio esquerdo; GM-d = Glúteo Máximo direito; GM-e = Glúteo Máximo esquerdo.

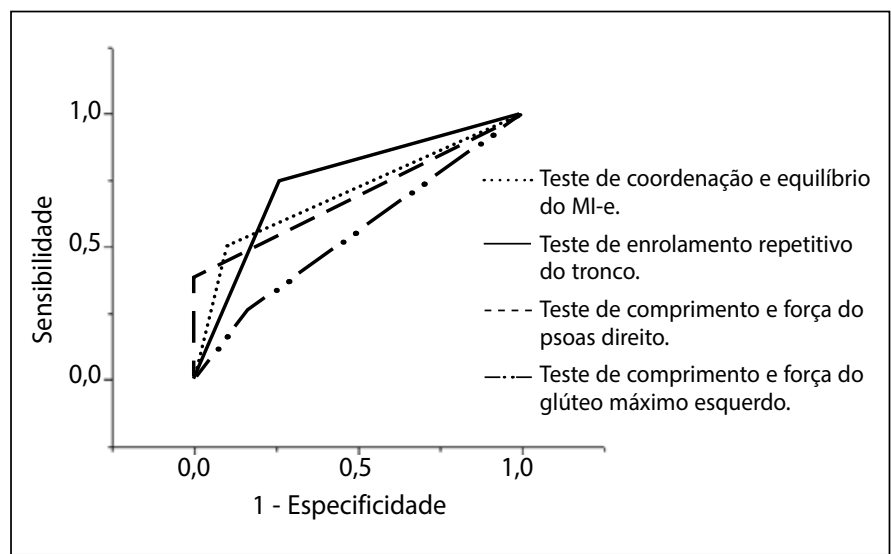

Figura 2. Curva Receiver Operating Characteristic (ROC) descrevendo a acurácia para o Teste de Coordenação e Equilíbrio do Membro Inferior esquerdo (MI-e), Teste de Enrolamento Repetitivo do Tronco, Teste de Comprimento e Força do Psoas direito e Teste de Comprimento e Força do Glúteo máximo esquerdo.

Tabela 1. Distribuição da frequência de ocorrências* de início de ativação muscular na condição de pré-ativação e alteração da pré-ativação no grupo total de voluntários e entre os gêneros.

\begin{tabular}{|c|c|c|c|c|c|c|c|c|}
\hline & $\begin{array}{l}\text { Total } \\
(\mathrm{n}=27)\end{array}$ & $\begin{array}{c}\text { Feminino } \\
(n=15)\end{array}$ & $\begin{array}{c}\text { Masculino } \\
(n=12)\end{array}$ & $c^{2} / P$ & $\begin{array}{c}\text { Total } \\
(n=27)\end{array}$ & $\begin{array}{c}\text { Feminino } \\
(n=15)\end{array}$ & $\begin{array}{c}\text { Masculino } \\
(n=12)\end{array}$ & $c^{2} / P$ \\
\hline Músculos & $\mathrm{ML}$ & $\mathrm{ML}$ & $\mathrm{ML}$ & & TrA/OI & TrA/OI & TrA/OI & \\
\hline Pré-ativação & $269(83 \%)^{* *}$ & $137(76 \%)^{* *}$ & $132(92 \%)^{* *}$ & $0,02 / 0,88$ & $203(63 \%)^{* *}$ & $83(46 \%)$ & $120(83,3 \%)^{* *}$ & $2,28 / 0,13$ \\
\hline$C^{2} / P$ & $77,8 / P<0,001$ & $13 / P<0,001$ & $40,7 / P<0,001$ & & $10 / P=0,001$ & $0,28 / P=0.58$ & $24,2 / P<0,001$ & \\
\hline Total & & 180 (100\%) & $144(100 \%)$ & & & $180(100 \%)$ & $144(100 \%)$ & \\
\hline
\end{tabular}

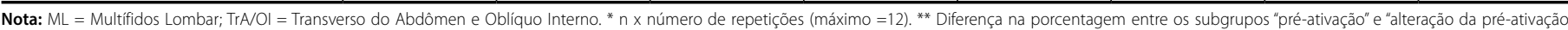
$\left(c^{2}=\right.$ teste de qui-quadrado, $\left.p \leq 0.05\right)$. ****iferenças entre gêneros $\left(c^{2}=\right.$ teste de qui-quadrado, $\left.p \leq 0.05\right)$. 


\section{DISCUSSÃO}

A hipótese inicial deste estudo foi parcialmente confirmada já que foram observados valores de sensibilidade, especificidade e acurácia adequados para os testes de coordenação e equilíbrio do membro inferior esquerdo e enrolamento repetitivo do tronco na detecção de alterações na atividade eletromiográfica de ativação da musculatura estabilizadora lombo-pélvica em voluntários assintomáticos para dor lombar. Nossos achados também demonstraram maior frequência de pré-ativação do que "alteração da pré-ativação" nos indivíduos assintomáticos em concordância com parâmetros sugeridos na literatura. Além disso, foi verificado um padrão de ativação diferente entre os gêneros, uma vez que foi verificada frequência significativamente maior de alteração na pré-ativação dos músculos ML e TrA/OI no gênero feminino.

O principal achado deste estudo foi que o teste de enrolamento repetitivo de tronco e o teste de coordenação e equilíbrio apresentaram acurácia moderada para detectar alterações na ativação muscular lombo-pélvica. Em 20 dos 27 voluntários avaliados ( $R O C=0,74$; sensibilidade $=0,75$ e especificidade $=0,73$ ), os resultados do teste de enrolamento repetitivo do tronco estavam em concordância com achados obtidos na ativação através da EMGS, ou seja, o teste de enrolamento de tronco mostrou-se dentro de valores de normalidade e foi observada pré-ativação através da EMGS. Esses resultados podem indicar que o teste de enrolamento repetitivo do tronco (que avalia a resistência dos músculos do abdômen) pode ser utilizado como parâmetro preditivo para detectar alterações no na ativação dos estabilizadores: TrA/OI e ML.

Não foram encontrados na literatura consultada estudos prévios que tenham verificado a acurácia de testes de capacidade funcional em relação a mensurações de ativação muscular. Entretanto, em estudo prévio, foram verificados bons níveis de acurácia de testes de resistência dos músculos do tronco em pacientes com dor lombar em relação a um grupo controle assintomático ${ }^{25}$. Estudos apontam que a resistência dos músculos abdominais em pacientes com dor lombar é menor do que em voluntários assintomáticos e a perda do controle muscular resultante da fadiga pode ser considerada uma das causas importantes de dor lombar ${ }^{4,26}$. Assim, a avaliação da resistência dos músculos do tronco parece ser um fator preditivo de dor lombar ${ }^{25}$.

Os músculos do tronco apresentam papel importante para estabilidade lombo-pélvica, de forma que uma menor resistência e força podem contribuir para o desenvolvimento de dor lombar ${ }^{13,27}$. Nossos achados demonstraram que o teste de enrolamento repetitivo de tronco apresentou moderada acurácia na detecção da alteração na pré-ativação para indivíduos assintomáticos. O teste de enrolamento repetitivo de tronco também pode ser chamado de teste de resistência/endurance dos músculos lombo-pélvicos. Redução no tempo de resistência durante o teste pode sugerir redução da performance da musculatura abdominal e menor ação estabilizadora da região lombo-pélvica. Como consequência, é possível o aparecimento de alterações na pré-ativação dos músculos lombo-pélvicos durante tarefas funcionais ${ }^{28}$. Desta forma, a acurácia moderada observada entre as duas medidas sugere que ambas parecem representar/detectar mecanismos de estabilização lombo-pélvica. Entretanto, os níveis apenas moderados de acurácia obtidos sugerem que uma medida não substitui a outra e, portanto, devem ser utilizadas em conjunto.

Apesar dos níveis adequados de acurácia $(0,69)$ e especificidade $(0,89)$ os valores de sensibilidade $(0,50)$ do teste de coordenação e equilíbrio do membro inferior, demonstram que o teste apresenta apenas boa especificidade (probabilidade de identificar os verdadeiros negativos, ou seja, probabilidade de identificar os indivíduos que realizaram à pré-ativação da musculatura estabilizadora lombo-pélvica através da EMGS). Os dados do presente estudo estão em concordância com as proposições prévias sobre a relação entre alterações de equilíbrio e estabilidade lombo-pélvica. Assim, como os ajustes antecipatórios são fenômenos dinâmicos, eles podem influenciar o equilíbrio, bem como estar ausentes em situações de redução do equilíbrio'.

Com relação à pré-ativação, embora a alteração no tempo de ativação muscular (feedforward) ocorra com maior frequência em indivíduos com dor lombar ${ }^{13}$, este estudo mostrou que indivíduos assintomáticos também podem apresentar alteração nesse mecanismo. Hodges et al. ${ }^{22}$ realizaram um estudo com oito voluntários assintomáticos, em que a atividade dos músculos do tronco foi avaliada com EMG de agulha durante o movimento rápido de flexão do ombro. Os resultados demonstraram que três voluntários não apresentaram pré-ativação do transverso do abdome em 70\% das repetições realizadas. Também concordando com nossos achados, Marshall e Murphy ${ }^{8}$ utilizaram a EMGS para avaliar a atividade dos músculos TrA/OI, oblíquo externo (OE) e reto do abdome (RA) de vinte voluntários assintomáticos, os resultados mostraram falta de pré-ativação do TrA/OI em quatro voluntários.

Em estudo prévio, foi avaliada a atividade do TrA/OI, OE, RA, ML e Eretor da Espinha (ES) pela EMGS de voluntários divididos em três grupos $^{28}$. No grupo controle e grupo com dor lombar e sem diagnóstico de instabilidade lombo-pélvica foi observada pré-ativação para os músculos ML e ES. Entretanto, no grupo de indivíduos com dor lombar e diagnóstico de instabilidade foi observada alteração da pré-ativação na maioria dos voluntários considerados ${ }^{28}$. Especificamente, o TrA/OI não pré-ativou na maioria dos voluntários durante as repetições nos três grupos ${ }^{28}$. Esses achados não estão em concordância com nossos achados, já que observamos maior frequência de pré-ativação do que de "alteração na pré-ativação" em sujeitos assintomáticos para ambos os músculos estudados (TrA/Ol e ML). É possível que diferenças nas características das amostras de ambos os estudos possam explicar as diferenças entre os achados. Silfies et al. ${ }^{28}$ consideraram sujeitos com o dobro da média de idade dos voluntários do presente estudo e com maior índice de massa corporal médio.

Dessa maneira, nossos achados dão suporte à tese de pré-ativação de músculos específicos do tronco em indivíduos assintomáticos (TrA/OI, ML) já que a frequência de pré-ativação dos participantes assintomáticos deste estudo foi de 83\% para o músculos ML e 63\% para o TrA/OI. Esses resultados confirmam a ação de estabilização dos músculos abdominais, ativando previamente ou em conjunto com os músculos do membro superior e possivelmente contribuindo para minimizar os momentos criados pela perturbação do membro superior?.

Em nosso estudo, foi observada diferença entre os gêneros no padrão de pré-ativação ou alteração da pré-ativação dos estabilizadores lombo-pélvicos. As mulheres demonstraram frequência significativamente maior de alterações na pré-ativação do TrA/OI e ML do que os homens. Há relatos prévios na literatura de maior ativação do músculo Ol nos homens do que nas mulheres, demonstrando dependência do gênero nas estratégias de ativação dos músculos abdominais'.

Neste estudo utilizamos uma bateria de testes divididos em quatro domínios: coordenação e equilíbrio, extensibilidade, resistência e comprimento e força. Os testes não foram realizados em ordem aleatória, porque assumimos que este procedimento representaria de forma mais fidedigna a prática clínica em que as avaliações clínicas são realizadas seguindo um roteiro pré-estabelecido em que a ordem dos testes é padronizada. Além disso, os testes mais vigorosos foram sempre realizados ao final do exame clínico. Este procedimento tem a vantagem de minimizar a chance de influenciar nas respostas comportamentais dos voluntários, quando, por exemplo, testes mais rigorosos/difíceis fossem realizados inicialmente. Em desenhos metodológicos em que a ordem do teste é escolhida de forma aleatória, é possível que um desempenho ruim do voluntário, possa influenciar no nível de adesão/disposição 
para realização dos demais testes subsequentes ${ }^{29}$. Por outro lado, uma limitação deste procedimento, é a impossibilidade de definir se a ordem dos testes foi passível de influenciar no desempenho do voluntário nos testes subsequentes. Contudo, o intervalo de 24 horas entre as avaliações físico-funcionais e o teste de movimento rápido de flexão do ombro (para coleta do sinal eletromiográfico) foi adotado, com o objetivo de minimizar possíveis efeitos de cansaço físico ou fadiga muscular.

Este estudo tem algumas limitações. Futuros estudos devem ser conduzidos em pacientes com dor lombar para verificar a acurácia do teste de rolamento repetitivo de tronco para detecção dos padrões de pré-ativação dos músculos estabilizadores lombo-pélvicos. Os valores de normalidade dos testes utilizados neste estudo não foram obtidos em estudos conduzidos na população brasileira, apesar de serem utilizados amplamente na literatura.

\section{CONCLUSÃO}

Foram observados valores de sensibilidade, especificidade e curva ROC adequados para os testes de Coordenação e Equilíbrio do membro inferior esquerdo e Enrolamento Repetitivo do Tronco na detecção de alterações na atividade eletromiográfica de ativação da musculatura estabilizadora lombo-pélvica em voluntários assintomáticos para dor lombar. Nossos achados também demonstraram maior frequência de valores adequados de pré-ativação do que "alteração da pré-ativação" nos indivíduos assintomáticos avaliados neste estudo. Além disso, foi verificado um padrão de ativação diferente entre os gêneros, uma vez que foi verificada frequência maior de alteração na pré-ativação dos músculos TrA/OI e ML no gênero feminino. Esses achados sugerem dependência do gênero nas estratégias de ativação dos músculos abdominais.

\section{AGRADECIMENTO}

Os autores agradecem a Fundação de Amparo à Pesquisa do Estado de São Paulo (FAPESP, 2008/01869-2) pelo apoio dado a esta pesquisa.

Todos os autores declararam não haver qualquer potencial conflito de interesses referente a este artigo.

CONTRIBUIÇÕES DOS AUTORES: Cada autor contribuiu individual e significativamente para o desenvolvimento do manuscrito. FJJ (0000-0001-8939-9078)*, RFNF $(0000-0001-9007-9274)^{*}$ e TCC (0000-0002-6222-4961)*, foram os principais responsáveis pela redação do manuscrito. FMA (0000-0002-4187-7058)*, NA (0000-0001$8001-301 X)^{*}$ e RGO (0000-0003-0904-9305)* realizaram a definição dos procedimentos de coleta com a eletromiografia, ajustes e elaboraram a rotina de análise do sinal eletromiográfico. FJJ realizou a coleta de dados e TDA (0000-0003-4473-026X)* realizou a tabulação dos dados e revisão. FJJ, RFNF e TCC realizaram a análise estatística. LCO (0000-0002-9853-7337)* e TDA realizaram a pesquisa e atualização bibliográfica. Todos os autores contribuíram com o conceito intelectual do estudo. *Número ORCID (Open Researcher and Contributor ID).

\section{REFERÊNCIAS}

1. Brooks C, Kennedy S, Marshall PW. Specific trunk and general exercise elicit similar changes in anticipatory postural adjustments in patients with chronic low back pain: a randomized controlled trial. Spine (Phila Pa 1976). 2012;37(25):E1543-50

2. Massé-Alarie H, Beaulieu LD, Preuss R, Schneider C. Task-specificity of bilateral anticipatory activation of the deep abdominal muscles in healthy and chronic low back pain populations. Gait Posture. 2015;41(2):440-7.

3. Lomond KV, Jacobs JV, Hitt JR, DeSarno MJ, Bunn JY, Henry SM. Effects of low back pain stabilization or movement system impairment treatments on voluntary postural adjustments: a randomized controlled trial. Spine J. 2015;15(4):596-606.

4. Ehsani F, Arab AM, Jaberzadeh S, Salavati M. Ultrasound measurement of deep and superficial abdominal muscles thickness during standing postural tasks in participants with and without chronic low back pain. Man Ther. 2016;23:98-105.

5. Morris SL, Lay B, Allison GT. Corset hypothesis rebutted-transversus abdominis does not co-contract in unison prior to rapid arm movements. Clin Biomech (Bristol, Avon). 2012;27(3):249-54

6. Pagé I, Nougarou F, Descarreaux M. Neuromuscular response amplitude to mechanical stimulation using large-array surface electromyography in participants with and without chronic low back pain. J Electromyogr Kinesiol. 2016;27:24-9.

7. Van Damme B, Stevens V, Perneel C, Van Tiggelen D, Neyens E, Duvigneaud N, et al. A surface electromyography based objective method to identify patients with nonspecific chronic low back pain, presenting a flexion related movement control impairment. J Electromyogr Kinesiol. 2014;24(6):954-64.

8. Marshall P, Murphy B. The validity and reliability of surface EMG to assess the neuromuscular response of the abdominal muscles to rapid limb movement. J Electromyogr Kinesiol. 2003;13(5):477-89.

9. Nelson-Wong E, Alex B, Csepe D, Lancaster D, Callaghan JP. Altered muscle recruitment during extension from trunk flexion in low back pain developers. Clin Biomech (Bristol, Avon). 2012;27(10):994-8.

10. Searle A, Spink M, Ho A, Chuter V. Exercise interventions for the treatment of chronic low back pain: a systematic review and meta-analysis of randomized controlled trials. Clin Rehabil. 2015;29(12):1155-67.

11. Davarian S, Maroufi N, Ebrahimi I, Farahmand F, Parnianpour M. Trunk muscles strength and endurance in chronic low back pain patients with and without clinical instability. J Back Musculoskelet Rehabil. 2012;25(2):123-9.

12. da Silva RA, Vieira ER, Cabrera M, Altimari LR, Aguiar AF, Nowotny AH, et al. Back muscle fatigue of younger and older adults with and without chronic low back pain using two protocols: A case-control study. J Electromyogr Kinesiol. 2015;25(6):928-36.

13. Hwang JA, Bae SH, Do Kim G, Kim KY. The effects of sensorimotor training on anticipatory postural adjustment of the trunk in chronic low back pain patients. J Phys Ther Sci. 2013;25(9):1189-92.

14. Massé-Alarie H, Beaulieu LD, Preuss R, Schneider C. Influence of chronic low back pain and fear of movement on the activation of the transversely oriented abdominal muscles during forward bending J Electromyogr Kinesiol. 2016;27:87-94.

15. Cox JM. Dor lombar: mecanismo, diagnóstico e tratamento. 6a. ed. São Paulo: Manole; 2002.

16. Liebenson C. Spinal stabilization - an update. Part 2 - functional assessment. J Bodyw Mov Ther. 2004;8(3):199-210.

17. Norris CM. Functional load abdominal training: part 1. Phys Ther Sport. 2001;2:29-39.

18. Vigatto R, Alexandre NM, Correa Filho HR. Development of a Brazilian Portuguese version of the Oswestry Disability Index: cross-cultural adaptation,reliability, and validity. Spine (Phila Pa 1976). 2007;32(4):481-6.

19. Kendall F, McCreary EK, Provance PG, Rodgers MM, Romani WA. Músculos provas e funções. 5a. ed. São Paulo: Manole; 2007.

20. Hermens HJ, Freriks B, Merletti R, Stegeman D, Blok J, Rau G, et al. European Recommendations for Surface ElectroMyoGraphy, results of the SENIAM project. Netherlands: Roessingh Research and Development; 1999. Disponível em: http://www.seniam.org/pdf/contents8.PDF

21. Gibson J, Mccarron T. Feedforward muscle activity: an investigation into the onset and activity of Internal oblique during two functional reaching tasks. J. Bodyw. Mov. Ther. 2004;8(2):104-13.

22. Hodges $P$, Cresswell A, Thorstensson A. Preparatory trunk motion accompanies rapid upper limb movement. Exp Brain Res. 1999;124(1):69-79.

23. Cowan SM, Bennell KL, Hodges PW. The test-retest reliability of the onset of concentric and eccentric vastus mediallis obliquos and vastus lateralis electromiographic activity in a stair stepping task. Phys. Ther. Sport. 2002;1:129-36.

24. Fischer JE, Bachmann LM, Jaeschke R. A readers' guide to the interpretation of diagnostic test proper ties: clinical example of sepsis. Intensive Care Med. 2003;29(7):1043-51.

25. Arab AM, Salavati M, Ebrahimi I, Ebrahim Mousavi M. Sensitivity, specificity and predictive value of the clinical trunk muscle endurance tests in low back pain. Clin Rehabil. 2007;21(7):640-7.

26. Villafañe JH, Gobbo M, Peranzoni M, Naik G, Imperio G, Cleland JA, et al. Validity and everyday clinical applicability of lumbar muscle fatigue assessment methods in patients with chronic non-specific low back pain: a systematic review. Disabil Rehabil. 2016:1-13.

27. Marques NR, Morcelli MH, Hallal CZ, Gonçalves M. EMG activity of trunk stabilizer muscles during Centering Principle of Pilates Method. J. Bodyw. Mov. Ther. 2013;17:185-91.

28. Silfies SP, Mehta R, Smith SS, Karduna AR. Differences in feedforward trunk muscle activity in subgroups of patients with mechanical low back pain. Arch Phys Med Rehabil. 2009;90(7):1159-69.

29. Luomajoki H, Kool J, de Bruin ED, Airaksinen O. Movement control tests of the low back; evaluation of the difference between patients with low back pain and healthy controls. BMC Musculoskelet Disord. 2008;9:170. 